\title{
Parameters of the average magnetic phase curves
}

\author{
V.D. Bychkov ${ }^{1}$, L.V. Bychkova ${ }^{1}$ and J. Madej $^{2} \dagger$ \\ ${ }^{1}$ Special Astrophysical Observatory, Russian Academy of Sciences, Nizhnij Arkhyz, Russia \\ email: vbych@sao.ru \\ ${ }^{2}$ Warsaw University Observatory, Al. Ujazdowskie 4, 00-478 Warszawa, Poland \\ email: jm@alkor.astrouw.edu.pl
}

\begin{abstract}
We analyse parameters of the average phase curves of the effective magnetic field $B_{\mathrm{e}}$ for 139 Main Sequence and other stars. 134 are chemically peculiar A and B type stars. This contribution presents the statistical properties of the fitting coefficients $B_{0}, B_{1}$, and $B_{2}$, and the distribution of the parameter $r=B_{\mathrm{e}}(\max ) / B_{\mathrm{e}}(\min )$.
\end{abstract}

Keywords. Magnetic stars, magnetic variability

\section{Introduction}

We have compiled all individual published measurements of the effective magnetic field $B_{\mathrm{e}}$ and have assembled a catalog of magnetic phase curves $B_{\mathrm{e}}(\phi)$ for all stars which exhibit a periodic variability of $B_{\mathrm{e}}$ with the rotational phase $\phi$. A total 139 stars were included in the catalog and 134 are Ap stars (Bychkov et al. 2004).

Most stars in the catalog exhibit periodic $B_{\mathrm{e}}$ variations, which can be satisfactorily approximated by a simple sine wave (first order term in the harmonic expansion). In some cases this is due to scarce or inaccurate $B_{\mathrm{e}}$ observations. However, 18 stars distinctly exhibit more complex $B_{\mathrm{e}}(\phi)$ curves, which we have fitted using also a second order harmonic term (double wave). The latter group of stars constitute about $13 \%$ of our sample. This lower limit may well increase when new average phase curves $B_{\mathrm{e}}(\phi)$ of higher accuracy become available.

The catalog presents 166 magnetic phase curves. Magnetic stars sometime exhibit quantitatively different phase curves when $B_{\mathrm{e}}$ values are measured by different methods or in different spectral lines.

\section{Parameters of magnetic variability: coefficients $B_{0}, B_{1}$, and $B_{2}$}

Fig. 1 shows the distribution of catalogued stars vs. coefficient $B_{0}$ for those stars in which phase curve $B_{\mathrm{e}}(\phi)$ was approximated by a sine wave. It does not include HD 215441 with its exceptionally strong $B_{\mathrm{e}}$ field, since this single point exceeds scale of the Figure. The average value of $B_{0}$ over all single-wave stars equals $21 \pm 138 \mathrm{G}$, the latter value representing the rather broad peak, and not the error.

Fig. 2 displays the analogous distribution of catalogued stars vs. coefficient $B_{1}$ (half amplitude of $B_{e}$ variability). This figure also shows only those stars which exhibit sine wave $B_{\mathrm{e}}(\phi)$.

There exists a large deficiency of stars with small half amplitudes $B_{1}$ of the effective magnetic field variations, see Fig. 2 . In the oblique rotator model small $B_{\mathrm{e}}$ variations can

$\dagger$ Present address: SAO RAS, Nizhnij Arkhyz, 369167 Russia. 


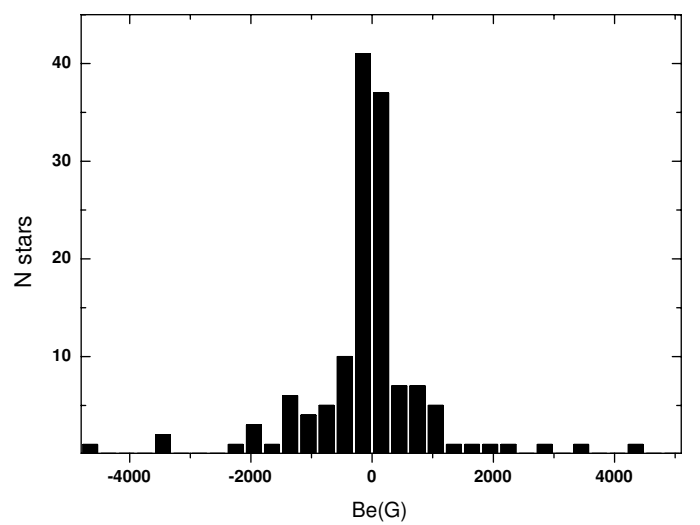

Figure 1. Distribution of catalogued stars vs. coefficient $B_{0}$ for those stars in which phase curve $B_{\mathrm{e}}(\phi)$ was approximated by a sine wave.

occur if the angle $\beta$ between the magnetic and the rotational axes is small or even zero. This observation suggests that magnetic stars avoid configurations with small angle $\beta$ angles.

Fig. 3 shows the distribution of stars vs. coeffcient $B_{0}$ for those stars, which exhibit magnetic phase curves with a double wave. The average value of $B_{0}$ equals $-473 \pm 296 \mathrm{G}$. Again, the number $296 \mathrm{G}$ represents broadening of the peak distribution. Only 18 stars contribute to this figure which results in poor statistics. However, stars which exhibit complex $B_{\mathrm{e}}$ phase curves (double waves) more frequently exhibit negative values of $B_{\mathrm{e}}$.

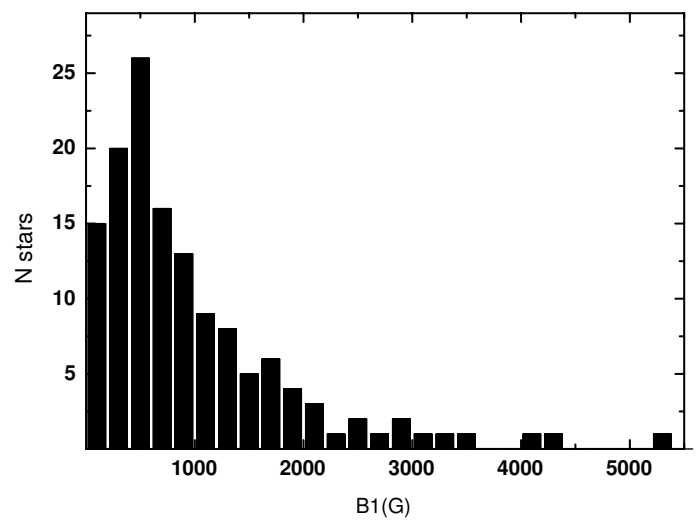

Figure 2. Distribution of catalogued stars vs. coefficient $B_{1}$ (semiamplitude of $B_{\mathrm{e}}$ variability). This figure also shows only those stars which exhibit a sine wave phase function. 


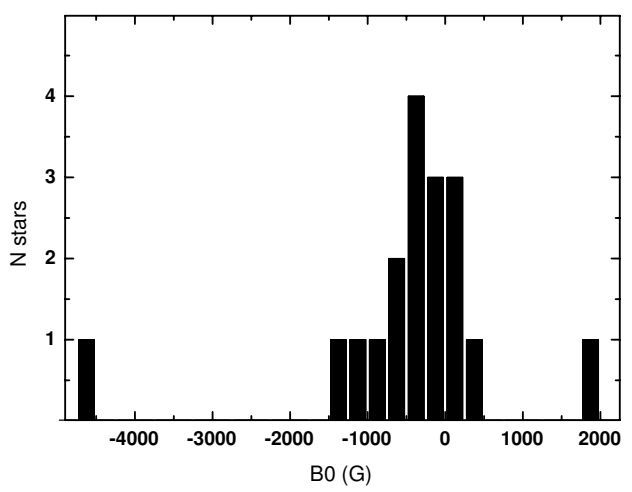

Figure 3. Distribution of stars vs. coeffcient $B_{0}$ for those stars which exhibit magnetic phase curves with double waves.

\section{Distribution of periods}

Fig. 4 is the distribution of stars in our catalog vs. the logarithm of period $P$, separately for all Ap stars with known periods (upper panel), and for stars with known phase curves (lower panel). The width of a single bin equals to 0.3 dex.

About $60 \%$ of all Ap stars have periods $P$ between 1 and 5 days. The same is true for stars with known phase curves.

\section{Distribution of the parameter $r$}

The parameter $r$ constrains the angle $\beta$ between the magnetic dipole axis and the rotational axis and the angle $i$ between the rotational axis and the line of sight (Stibbs 1950)

$$
r=\frac{\cos \beta \cos i-\sin \beta \sin i}{\cos \beta \cos i+\sin \beta \sin i}
$$

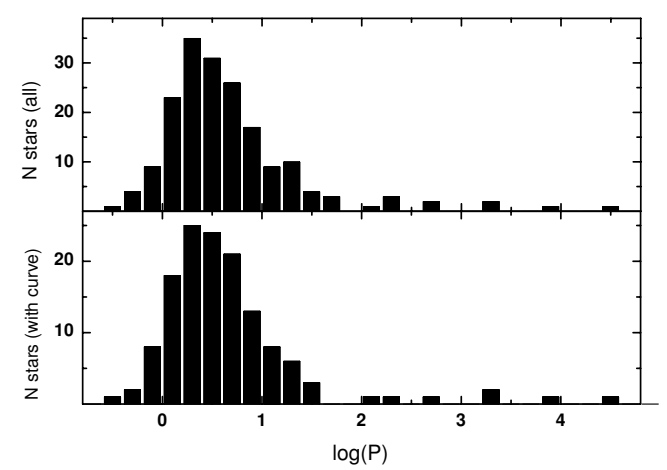

Figure 4. Distribution of stars in our catalog vs. the logarithm of period $P$, for all Ap stars with known periods (upper panel), and for stars with known phase curves (lower panel). 


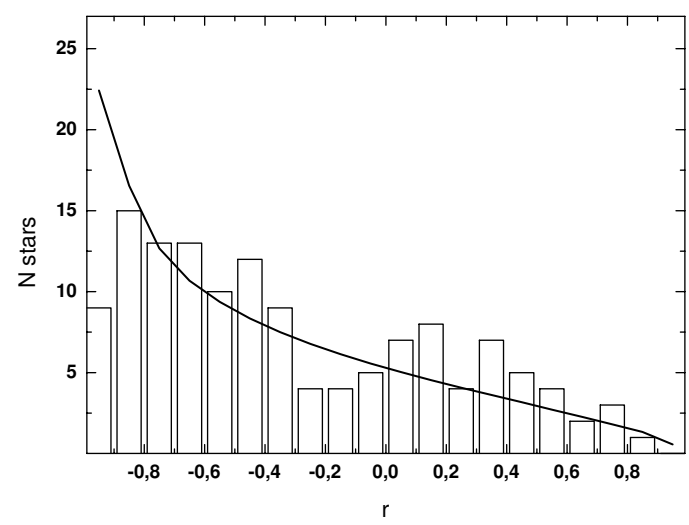

Figure 5. Measured distribution of the parameter $r$ vs. theoretical distribution, the latter corresponding to random $\beta$ and $i$ angles.

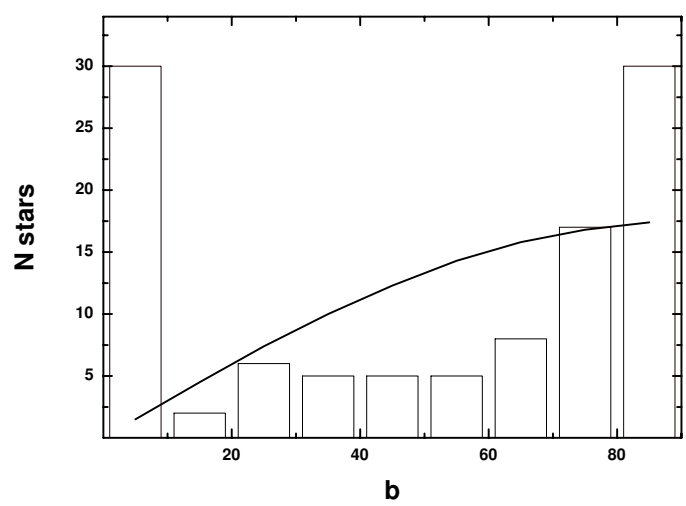

Figure 6. Distribution of stars in our catalogue vs. the angle $\beta$ between magnetic and rotational axes.

One can express this parameter by observed quantities

$$
r=\frac{B_{\mathrm{e}}(\min )}{B_{\mathrm{e}}(\max )} .
$$

Fig. 5 shows the distribution of stars in our catalogue vs. the observed parameter $r$, see the enclosed histogram. The solid line is a theoretical distribution obtained using the assumption that the angle $\beta$ is uniformly distributed. This figure demonstrates substantial differences between the observed and theoretical distributions of the parameter $r$.

Finally, we attempted to collect or to recompute the distribution of the angle $\beta$ in the catalogued stars, based on our collection of phase curves $B_{\mathrm{e}}(\phi)$. Such a distribution is shown in Fig. 6. There are an excess of stars with angle $\beta \rightarrow 0$, which contradicts previous results obtained in Fig. 5. 


\section{Summary}

We compiled and collected in the single catalogue all available magnetic phase curves $B_{\mathrm{e}}(\phi)$, and expressed them in the uniform manner. The catalog lists phase curves for 139 stars. 134 of which are magnetic Ap stars. This catalog permitted us to find all the parameters of stellar magnetic variations and to perform homogeneous analyses.

We note the apparent deficiency of stars with small variations of $B_{\mathrm{e}}$, see Fig. 2. Also there exists a deficiency of stars with small and intermediate values of the parameter $r$, i.e., in particular at the limit $r \rightarrow-1$, as compared with the theoretical model of uniform distribution of the angle $\beta$.

\section{Acknowledgements}

Our research is based on data compiled and posted in the SIMBAD, ADS, and CDS databases. We acknowledge support from the Polish Committee for Scientific Research grant No. 1 P03D 00126.

\section{References}

Bychkov, V.D., Bychkova, L.V., Madej, J. 2004, A\&A in press

Stibbs, D.W. 1950, MNRAS 110, 395 